\title{
On the Possibility of Mandarin Chinese as a Lingua Franca
}

\author{
Christopher Plumb (Corresponding author) \\ Abu Dhabi National Oil Company Technical Institute \\ P.O. Box 898/13 (ADNOC ATI), Abu Dhabi, United Arab Emirates \\ Tel: 971-52-948-3277Ｅ-mail: cplumb@adnoc.ae
}

Received: May 15, 2016 Accepted: June 14, 2016 Published: June 15, 2016

doi:10.5296/jei.v2i2.9458 URL: http://dx.doi.org/10.5296/jei.v2i2.9458

\begin{abstract}
Recently there has been an increase in the number of students learning Mandarin Chinese (in mainland China and internationally). This increase has led to speculation that Mandarin Chinese is becoming a mainstream global language to the point of becoming a lingua franca. This paper utilizes research findings from different regions and focal points and argues that Mandarin Chinese could be accepted as a lingua franca within some contexts, but is unlikely to do so in others. It argues that Mandarin Chinese is generally accepted as the lingua franca of China and a possible lingua franca within the East Asian region, while unlikely to become a lingua franca globally. The paper compares a number of different reasons for studying Mandarin Chinese by different stakeholders (i.e. governments, school boards, individual students) as well as comparative numbers of language learners. This paper also examines if Mandarin is the dominant and undisputed form of global Chinese. It concludes by demonstrating that there is a greater need for further research into Mandarin Chinese as a lingua franca.
\end{abstract}

Keywords: Mandarin Chinese, lingua franca, EAL, CAL, Confucius institutes, Putonghua

\section{Introduction}

Throughout history, languages around the world have become foremost within their spheres of influence. They are often replaced over time by other languages as the new language becomes more dominant (Ning, 2010). When a language becomes so dominant that it is a means of communication between peoples who speak different languages, it becomes a lingua franca. Within the last two hundred years, English has surpassed all other languages in global use and has become the world's de facto lingua franca. Other languages that have similar global impact include Spanish, French, German and Mandarin Chinese. A noteworthy 
study performed by David Graddol (1997), suggested that Mandarin Chinese will become one of the world's top languages by 2050 (in Gil, 2011: 52). Graddol's study was commissioned as a result of the increasing global importance of China, both economically and culturally. Several researchers have argued, to varying degrees, that Mandarin Chinese is growing in influence and is becoming an important international language (Lo Bianco, 2007; Chmelynski, 2006; Ding \& Saunders, 2006; Zhang \& Li, 2010; Zhao \& Huang, 2010; Dretzke \& Jordan, 2010; Gil, 2011; Kirkpatrick, 2011; Lu, 2008; Zhang, 2011; Hartig, 2012; Hua \& Wei, 2014).

While researchers tend to agree that English is the current global lingua franca and will be for some time, several researchers suggest that Mandarin Chinese is already or will become a lingua franca in the future. However, the degree to which Mandarin will become a lingua franca is disputed. Many argue that Mandarin will become a lingua franca amongst ethnic Chinese people outside of China or specifically within China (Lo Bianco, 2007); others argue that it could become a lingua franca for all of East Asia (Ding \& Saunders, 2006), some argue that Mandarin could replace or at least compete equally with English (Lu, 2008; Smith, 2005; Zhang, 2011; Zhao \& Huang, 2010). This paper analyses relevant studies written or translated into English with respect to Mandarin Chinese as a lingua franca within the People's Republic of China (PRC) and the East Asian cultural region, as well as its global use to determine its feasibility as a lingua franca. It will argue that Mandarin will be cemented as the lingua franca within China in the near future, it may compete with but not surpass English as a lingua franca in the East Asian cultural region and, finally, is unlikely to become a worldwide lingua franca. Prior to that, the concept of lingua franca will be examined as well as Mandarin Chinese as a defined language.

\section{Lingua Franca and Mandarin Chinese Defined}

One of the issues with discussing Mandarin as a lingua franca is in defining Mandarin Chinese and lingua franca. Alan Firth (1996), in his definition of English as a lingua franca, defined it as 'a 'contact language' between persons who share neither a common native tongue nor a common culture, and for whom English is the chosen foreign language of communication" (in Kirkpatrick, 2011: 213). Using this definition of lingua franca as a concept, it becomes useful to break down the criteria further so the concept can be readily understood in the uncertain context of Mandarin Chinese.

A lingua franca as a 'contact language' between people who do not share a common language could be an acceptable definition for a regional lingua franca. David Crystal (1998; 2006) proposed a three-pronged set of criteria to judge whether or not a language is a global lingua franca. He stated that a language must 1) be a native language of a particular state or region, 2) be widely adopted as an official language elsewhere, and 3) be a priority in language teaching around the world (in Gil, 2011: 53). A regional lingua franca, therefore, can be seen as having different criteria than a global lingua franca. It is unfortunate that studies on the subject do not readily introduce these distinctions; if not properly defined this can cloud any judgment as to whether or not Mandarin Chinese is a lingua franca. It is important that relevant literature defines exactly what criteria it utilizes when making an 
assertion that a language is a lingua franca.

Mandarin Chinese is one of seven major Chinese dialects, which by itself could be treated as a single language or several different independent languages due to its many sub-varieties (Gil, 2011). The most common form of Mandarin is Putonghua, a modernized form of Mandarin, which borrows from several sub-dialects of Mandarin and has been painstakingly developed by the government of the People's Republic of China (PRC) since the 1950's (Li, 2006). It is this form of Chinese that has been adopted within China and has been rapidly spreading outside of China for the last thirty years. This form, if any, would be the proposed form of Chinese if it were to become a lingua franca. It is partially because of the fragmentation of Chinese as a language group in general that prevents one version, in this case Putonghua, from becoming an easily accepted lingua franca. With this understood, an examination of Mandarin Chinese as a lingua franca in different contexts can now be undertaken.

\section{Mandarin Chinese as a Lingua Franca in China}

Although Han Chinese form the majority of the population of China (92\%), China has 55 minority groups officially registered within its borders (Gao \& Smyth, 2011: 342). The government of the People's Republic of China (PRC), along with prior ruling bodies throughout history, have long striven to achieve unity within China, often through the means of language policy. Unification of people through language within China has never been an easy task. Minority groups as well as large sections of the Han Chinese population speak languages other than Mandarin. From a governmental perspective, the promotion of Mandarin Chinese as a lingua franca within greater China, through its modern derivative, Putonghua, has been a priority (Lo Bianco, 2007; Li, 2006; Lu, 2008).

With respect to Mandarin as a lingua franca within China, after a slow start to produce research, the results appear to agree. In 2007, Joseph Lo Bianco argued that too few studies existed on language policy within China written in Western languages, such as English. Since then, several detailed and original studies have been published, often citing each other. This lends to their credibility as they do not often contradict each other. Along with Lo Bianco's landmark study on Chinese language policy, which is cited by several researchers in this study, Gao and Smyth's (2011) study on the economic returns for internal migrants within China, as well as Li's (2006) study on Chinese as a lingua franca within greater China afford a solid foundation from which to compare other studies. All three studies and aspects of other research agree that Putonghua has been aggressively promoted by the government of China and has made considerable steps towards being accepted by non-Mandarin speakers throughout China, though to different degrees (Ding \& Saunders, 2006; Kirkpatrick, 2011; Zhao \& Huang, 2010).

Although the three major studies by Lo Bianco, Gao, and Li rely heavily on statistics provided by the Chinese government and sometimes from government newspapers (which could be biased), they appear to be well researched and show similar conclusions. This contrasts with the results of studies examining Mandarin as a lingua franca within East Asia; studies in this area often disagree. One aspect where studies within the PRC differ slightly 
relates to problems the PRC has had in overall acceptance of Putonghua from all areas and peoples within the country. Though acceptance has been widespread, it has been far from uniform. In their study of migrants, Gao and Smyth (2011) argued that those who can either speak or comprehend Putonghua have a greater chance of finding employment with higher wages than their counterparts who lack comprehension in Putonghua. They argue that a leading cause for acceptance of Putonghua is for socioeconomic reasons. They also note that children appear to have accepted Putonghua more rapidly than their parents as a result of being placed in state run schools (Gao \& Smith, 2011). Alternately, Li suggests that some areas of China are unwilling to accept Putonghua en masse as their particular Chinese language is considered a high prestige dialect (2006). His discussion of problems relating to Cantonese speaking areas of China (as well as the economic and cultural influence from Hong Kong) is well supported by empirical evidence and is a subject which is also addressed by other researchers (Li, 2006; Lu, 2008; Gao, 2012).

Since there are approximately 250-350 million Chinese citizens learning English as an additional language, Dan $\mathrm{Lu}$ asserts that even though Mandarin is used within the government, schools, industry, army, and other areas, individuals outside of these constraints (or even within them) may use English as their common language (2008). Lu's study notes that there has been considerable growth in English language education within China, but often outside of the control of the state apparatus. This finding is rarely mentioned by other researchers and leaves unanswered questions in relation to Putonghua and its acceptance within China. Nevertheless, considering seventy percent of Chinese citizens can understand and fifty percent can speak Putonghua within greater China (Li, 2006), this is one area where Mandarin could be considered a lingua franca.

\section{Mandarin Chinese as a Lingua Franca in East Asia}

For centuries Chinese culture and language have been dominant in East Asia when compared to other cultures and languages. Beginning in the late 1700s, western influence in the region diluted Chinese influence to the point that these two spheres of influence coexist today. Several nations within the region contain either ethnic Chinese majorities (e.g., Taiwan, Singapore) or substantial minority populations (e.g., Malaysia, Korea, Japan, and Myanmar). These ethnic enclaves, however, do not necessarily speak Mandarin Chinese. With this in mind, it becomes more problematic to discuss Mandarin as a lingua franca within this region. In addition, there is a lack of research produced specifically on Chinese as a lingua franca within the East Asian region. Instead, research tends to focus on specific examples, especially relating to Singapore, Taiwan, Hong Kong or Macao (Bokhorst-Heng, 1999; Scott \& Tiun, 2007; Wee, 2009; Zhao \& Liu, 2010; Gao, 2012; Kobayashi, 2013).

Through discussion of Singapore's Speak Mandarin campaign (SMC) and Taiwan's Mandarin only policy, research highlights some of the problems within the Chinese language itself that make its acceptance as a lingua franca within the region difficult. Singapore's leaders noticed that its Chinese population's language was fragmented among dialects and introduced Mandarin over a thirty year period with the goal of creating a homogenous Chinese-speaking population; the campaign, however, has been problematic in its acceptance for similar 
reasons as within the PRC (Lo Bianco, 2007; Bokhorst-Heng, 1999; Li, 2006; Wee, 2009). Challenging the SMC initiative were the dual problems that Singapore inherited a society built on English colonialism and that it consists of three major ethnic groups (Chinese, Malay and Indian/Sri-Lankan) often using English as a local lingua franca, impeding Chinese use (Wee, 2009). Studies discussing Singapore and the SMC campaign have widely cited both quantitative and qualitative investigations, giving sources on the Singapore specific aspect considerable weight (see Bokhorst-Heng, 1999 for a particularly good example).

Taiwan, for unique reasons, has become a complicated component of this situation. As Taiwan was colonized by non-Mandarin speaking coastal Chinese groups starting in the 1500s, and later the Japanese, until 1945 Taiwan had limited historical use of Mandarin. Only after the Chinese civil war (ending in 1949) did the nationalist Chinese under the Kuomintang institutionalize a Mandarin only policy that was quite strict and led to the adaptation of Mandarin by ninety percent of the population by 1987 (Li, 2006; Scott \& Tiun, 2007). In recent times, however, Taiwan has begun to assert its own identity as one separate from mainland China. As a means to cultivate a unique Taiwanese identity, southern Min (a dialect of Chinese long established in the southern portion of Taiwan) has been added to Mandarin to create a hybrid language known locally as Taiwanese (Li, 2006). This meant that, although Taiwanese is mutually intelligible with Putonghua, Taiwan as a nation has endorsed a different version of Chinese than mainland China. Additionally, many Taiwanese learn Cantonese as a second language, owing to economic ties with Guangdong province in mainland China, Hong Kong and Macao, though more empirical data is required to understand the total effect of this trend on the population (Scott \& Tiun, 2007).

Hong Kong and Macao represent two areas where Mandarin Chinese has never been the dominant language. Cantonese dialect speaking areas originally, later mixed with English and Portuguese through colonization, these two enclaves have had a separate modern history and economic development than mainland China. Though in both cases, the study of Putonghua as a second language has been on the rise and larger portions of the populations interact through its use, Cantonese remains the dominant language (Lo Bianco, 2007; Li, 2006). In addition, according to Lo Bianco's (2007) research, it appears that the upper classes in Hong Kong and Macao are learning both Mandarin and English to become what he terms, "elite bilinguals", ostensibly for economic reasons. This is in contrast to the lower classes, which have resisted learning Mandarin as they see it as a lower class language when compared to Cantonese due to their interaction with recently arrived mainland immigrants of lower socioeconomic status (Lo Bianco, 2007). There are few studies that make note of this trend; it is clear that more research must be done to ascertain the validity of Lo Bianco's claim. Gao (2013) noted that local instructors in Hong Kong teaching South Asian minority students taught Cantonese and considered themselves to be cultural and linguistic trainers to their new students of Chinese. The strong use of Cantonese instruction as a means to preserve the local dominant culture would be in conflict with the development of Putonghua as a dominant language.

As mentioned earlier, there is a lack of research focusing on the region as a whole and its acceptance or use of Mandarin. One study that does examine a large area of the East Asian 
cultural area is Andy Kilpatrick's (2011) conference speech about the Association of South East Asian Nations (ASEAN) nations and their acceptance of English as an official lingua franca for that group of 10 Southeast Asian nations. Though brief and lacking in documentation, Kilpatrick notes that English has been officially accepted as the lingua franca for the economic and cultural group, and that only Singapore has a majority Chinese population amongst group members (Kirkpatrick, 2011). It is asserted that the majority of second language learners in the region are studying English and not Chinese.

Information relating to other countries in East Asia and their acceptance of Mandarin is lacking and thus a clear picture remains elusive. Generally, it is limited to the amount of students studying Mandarin, which is often compared with statistics relating to European languages (including English), rather than other Asian languages, such as Japanese. In every case, English is studied more frequently, especially in countries such as the Philippines (where it is an official language), Korea, Japan and Thailand. That said, there has been a slight shift towards Asian language study in Asian universities, but it remains doubtful Chinese study will grow beyond the study of English. Kobayahsi (2013) found that in Japan, although Chinese study was on the rise compared to European languages such as German and French, it did not surpass the study of English. It remains clearly discernable that unlike in mainland China, Mandarin would face considerably greater difficulties becoming a lingua franca in East Asia, especially in light of the dominance English already enjoys there.

\section{Mandarin Chinese as a Global Lingua Franca}

The growth of Mandarin Chinese language and culture globally has been acknowledged in multiple forms. However, the extent to which Mandarin is on the rise remains unclear. Many studies cite newspapers, popular publications and magazines as proof that demand for Chinese language skills is increasing at an exponential pace (Lo Bianco, 2007; Chmelynski, 2006; Lu, 2008; Zhang, 2011; Zhao \& Huang, 2010; Zhang \& Li, 2010). Ning (2010: 59) went so far as to term the phenomenon, "Chinese fever". Additionally, many of the studies provide data with respect to the number of foreign Mandarin language learners internationally or within China, but these statistics often do not disseminate the reasons why people are studying Mandarin or identify those who are studying it. An illustrative example of this can be found when Jeffrey Gil states that forty million people worldwide are studying Mandarin, but that the number includes the many ethnic Chinese who are learning the language abroad as a means to reclaim their heritage (2011: 54). In line with this reasoning, Dan Lu argues that Mandarin Chinese is used in various countries but usually between Chinese ethnic groups; early emigrants were often formerly from coastal regions, speaking Cantonese or Hokkeinese for example. He reasons that Mandarin is not often used for cross-racial communication to the same extent that English or Spanish is (Lu, 2008: 270, 274). Dretzke and Jordan (2010) appear to agree with these findings in their study of students from the United States, noting that Chinese students formed a large group interested in cultural ties, while acknowledging that other groups primarily indicted economic reasons as their rationale for learning Chinese; in that study $20-25 \%$ of the students were White North Americans compared to $75-80 \%$ Asian. 
Even if a significant percentage of Chinese as a foreign language students are ethnically Chinese, the forty million Mandarin students worldwide mean that a considerable number of non-Chinese people are learning the language. Researchers tend to agree that the impetus behind this is threefold and centered on the initiatives of the government of the PRC. The threefold approach by the PRC is anchored in the National Office for Chinese as a Foreign Language (NOCFL), the Hanyu Shuping Kaoshi (HSK - the Chinese as a foreign language proficiency test) and the Confucius Institutes (institutions set up and funded in foreign countries on behalf of the PRC government to further Mandarin language education). The Confucius institutes constitute the nexus of the three and as of 2009 there were 339 institutes in 83 countries (Zhao \& Huang, 2010: 129). Considering the first institute was established in 2004 (in Seoul, South Korea) and the stated NOCFL goal is to reach 500 by 2020, this should be seen as a noteworthy development in such a short span of time. Working together with local institutions, the Confucius institutes have played a key role in the expansion of Mandarin language education globally (Lo Bianco, 2007).

Important to this, however, is that the development of these PRC government establishments have not been without controversy. According to Hongqin Zhao, the Swedish Riksdag (parliament) and the Canadian Security and Intelligence Service (CSIS) have raised concerns that the Confucius institutes are possible vehicles for PRC propaganda (2010). Hartig (2012) voiced similar findings in his study of Confucius institutes in Germany. Many other researchers have argued parallel concerns with the direct influence the PRC has with respect to Mandarin language education. One specifically notes the case of Vietnam, which has been wary of the initiatives, citing concerns related to linguistic imperialism due to its recent history with respect to Russian and English (Ding \& Saunders, 2006). More recently, Zhang and Adamson (2015) argued that the Confucius institutes have become viewed with a certain amount of consternation in the United States as a result of the PRC government's involvement in their operation. In the United Kingdom, Pérez-Milans (2015) found that the study of Putonghua was rising and that more schools were offering classes in conjunction with the Confucius institutes. The study centered on the case of one school that transformed itself into a "language specialist school" in conjunction with a Confucius institute, leading to differences of opinion and a buildup of concerns.

Conversely, a small study produced by Zhang (2014) suggests that it is important to learn Chinese within a cultural context that can be provided through the guidance of the PRC and the Confucius institute context that is often lacking in other teaching environments. Hua and Wei (2014) noted a similar finding when reviewing strategies employed at Confucius institutes and classrooms overseen by the Chinese government. Overall though, government influence can hamper the effort for Mandarin Chinese to become a global language in ways that do not exist to the same degree for English or Spanish for example, as in many cases it appears to be an externally guided effort, at times viewed with suspicion as to its motives.

Assuming that the PRC is not a major influence on the decisions of people studying Mandarin, statistics show that Mandarin growth is significant, but it is often compared in a vacuum. This is generally done by utilizing statistics from the past that displayed little growth in Mandarin language learning when compared to recent developments. Because the PRC has 
only recently opened up (in the last thirty years), language learning growth generally started slowly, followed by seemingly exponential growth. Much of the research cites fantastical and varying statistics that express a rapid growth of Chinese language learning: 400000 students took the HSK in 2005; 40 million studying Mandarin in 2005; more than 25\% of American universities (800) now offer Chinese programs; Mandarin is one of 6 official languages at the UN; double digit growth since the 1970's; 50,000 K-12 students in the United States studying Mandarin, a 52\% increase since 2002; 10\% of internet sites are in Mandarin; 200000 foreign students in China studying Mandarin.

These statistics, reproduced time after time are certainly impressive. Many come from studies arguing that Mandarin could one day compete with English as a global lingua franca (Lu, 2008; Smith, 2005; Zhang, 2011). These findings and statistics are not often compared to the numbers of students studying other languages. This is problematic because proof of language popularity rests in comparative statistics related to English and other languages. Ding and Saunders stated that in 2006, in the United States alone, there were 24,000 students studying Chinese (which does not correspond with the above statistics) while 1,000,000 students were studying French (2006). Clearly many students chose French over Chinese; it should be noted that Spanish numbers may be even higher. Another example is the number of students worldwide: generally it is accepted among researchers that almost 400 million people worldwide are studying English while there are 40 million studying Chinese. Essentially ten times as many people study English when compared to Mandarin Chinese, assuming the statistics are accurate. Finally, Gil argues that much of this is inconclusive, since these findings are somewhat incomplete because empirical and statistical evidence varies for different and complex reasons; this notion was also argued by Lu (Gil, 2011; Lu, 2008).

If one were to acknowledge that Mandarin is rapidly growing in its own right, outside of evidence related to other languages, it still faces one truly significant hurdle to becoming a lingua franca, oddly one that is not touched upon by many researchers. Smith (2005) argued that in order for a language to become a proper global lingua franca it must meet six criteria. One of these, and according to him the most important one, is that the language should be easy to learn (2005). With respect to Mandarin Chinese, Lu (2008) took the most forthright approach to this problem, breaking down the many ways in which Mandarin could be considered as more difficult to learn on average than other languages. In his detailed study, he notes that the Chinese alphabet is based on pictographs, of which 2000 must be learned to achieve near proficiency; there is a major disconnect between spoken and written forms of the language; even the pinyin version of Mandarin using Romanized characters for computer use does not mesh well with the standard characters ( $\mathrm{Lu}, 2008: 270-274)$. He concludes that it is unlikely that Mandarin will become a significant global language because it is too difficult to write for additional language learners when compared to alternatives and that its use will be mostly within international ethnic-Chinese communities. Lin, Huang and Chen (2014) also noted in a study of computer assisted language learning in the United States, that the availability of new electronic resources and related pedagogy was an impediment to the growth and development of Mandarin Chinese. This was due to the lack of rigorous educational resources produced. Though it seems unusual that other researchers failed to 
mention the difficulty of learning Mandarin, and some would argue that discussing relative levels of difficulty is subjective, the argument made by the few studies that addressed this concern is important and requires attention.

Clearly from the research it can be seen that making a case for the rapid growth of Mandarin Chinese or its ability to become a global language or even the world's lingua franca is too complex to ascertain with any degree of certainty. This is because it is not fully accepted within the PRC, despite continuous promotion by the government. It has not been accepted fully due to widespread use within the PRC of other forms of the Chinese language family and the diverse culture of local regions which try to protect their indigenous language. Furthermore, outside of the PRC, a convoluted situation has arisen whereby the different countries and regions of East Asia have their own perspective on the Chinese language, therefore making it incredibly difficult for Mandarin Chinese to prevail as a lingua franca. What is clear is that most researchers state that English at present is the world's lingua franca and that this will not change in the immediate future because its use is so globally widespread. It is also clear that if Mandarin Chinese were to become a global lingua franca, it would have to achieve even more rapid growth in conjunction with a significant decrease in people learning English as well as other popular languages. Therefore, Mandarin Chinese cannot be seen as a global lingua franca.

\section{Suggestions for Further Research}

With reference to some of the already noted gaps in the research and suggestions therein, it is important to suggest more clearly, possibilities for further research in addition to some suggestions not already made. One major area of research that should be considered is evidence gathering related to the growth of Mandarin Chinese and the claimed exponential increase in demand for Chinese language, or alternatively, fears from representatives from other language groups about Mandarin's rise as a global language. In particular, Zhang's study cites several different sources related to the rise of the Mandarin language. These sources often rely on newspaper stories, magazine articles and news reports, especially those published in the PRC (Zhang, 2011). Many of these sources of evidence are scarcely scholarly and thus their validity could be called into question. More scholarly research should be performed to investigate the claims of those who feel Mandarin is truly becoming a global language or indeed a lingua franca in any context. Lu argues that the interest in Mandarin, "is only a bubble that will burst in the end" ( $\mathrm{Lu}, 2008: 269)$. This is not to dispute the nature of the claims completely; clearly evidence from other studies does indeed show a significant rise in Mandarin Chinese language use globally, but the reasons for and the nature of its rise compared to other languages must be studied in greater detail. In order to demonstrate if the uptake of Mandarin Chinese is as prevalent as stated by the authors of many studies already published in this area, much more detailed and quantitative research has to be carried out. This has to be undertaken with respect to Mandarin Chinese growth independently and in relation to other language development, such as that of English.

Alternatively, the nature of the government of the PRC's involvement in the promotion of Mandarin language should be considered more carefully. Studies should be undertaken to 
determine, if possible, the overall impact of the involvement of the PRC on Mandarin growth. As Mandarin growth seems directly related to the efforts of the Chinese government, this should be considered important. Related to this would be research comparing the PRC influence to other governments' involvement in the promotion of their own languages, such as The British Council on behalf of the United Kingdom. Only then could claims of PRC governmental influence be understood in a useful manner.

Finally, the quantitative evidence displayed in the articles covered in this review does not emphasize which specific countries are engaged in learning Mandarin. English is generally learned uniformly across the world. In contrast to this, for example, of the 339 Confucius institutes worldwide, most are located in what is known formally as the "first world"; 70 of which are located in the United States in addition to 35 in the United Kingdom (Zhao \& Huang, 2010). This means that about one third of the institutes are located in just two countries, which does not allow for a uniform distribution of instruction. For example, Mandarin Chinese instruction in Germany is not widespread and one study found that more than half the people in Germany surveyed had a negative perception of China (Ding \& Saunders, 2006; Hartig, 2012). It would be useful to know exactly where Mandarin is growing significantly and where its growth is limited. This would show if Mandarin Chinese has the real potential to become a global lingua franca. With respect to the difficulty of the language as discussed by $\mathrm{Lu}$, it would also be noteworthy to examine which language groups worldwide are more compatible with Mandarin as an additional language if there is any significant disparity at all.

\section{Conclusion}

An analysis of various studies relating to the global use of Mandarin Chinese can demonstrate the importance of Mandarin Chinese within different contexts. Though a complete picture of Mandarin Chinese as a lingua franca remains elusive, concrete conclusions can be made that demonstrate how the language is accepted in different contexts. Within greater China and the international ethnic Chinese community, Mandarin Chinese is an increasingly used language and is therefore important. On a global stage, however, Mandarin Chinese cannot be seen as being a true lingua franca, despite statistics outlining its escalating importance. The research required to fully understand modern trends in Mandarin Chinese language use is quite complex and at present incomplete. Only with further research into use of Mandarin Chinese identified by this and other studies, can the global impact of Mandarin Chinese language use be fully understood.

\section{Acknowledgements}

Thank you to Dr. Julie Kerekes, University of Toronto; Carrie Demmans Epp, University of Toronto; Dr. Kate O’Sullivan, Al Shohub Private School.

\section{References}

Bokhorst-Heng, W. (1999). Singapore's speak mandarin campaign: Language ideological debates in the imagining of the nation. In J. Blommaert (Ed.), Language ideological debates (pp. 235-266). Berlin: Mouton de Gruyter. http://dx.doi.org/10.1515/9783110808049.235 
Chmelynski, C. (2006). Teaching Chinese as tomorrow's language. The Education Digest, 71(6), 59-63.

Crystal, D. (2006). English worldwide. In R. Hogg \& D. Denison (Eds.), A History of the English Language (pp. 420-439). Cambridge: Cambridge University Press. http://dx.doi.org/10.1017/CBO9780511791154.010

Ding, S., \& Saunders, R. (2006). Talking up China: An analysis of China's rising cultural power and global promotion of the Chinese language. Springer Netherlands. http://dx.doi.org/10.1007/s12140-006-0021-2

Dretzke, B. J., \& Jordan, K. (2010). Expanding secondary school Chinese language programs: A study of potential challenges. American Secondary Education, 38(2), 57-72.

Gao, F. (2012). Teacher identity, teaching vision, and Chinese language education for South Asian students in Hong Kong. Teachers and Teaching, 18(1), 89-99. http://dx.doi.org/10.1080/13540602.2011.622558

Gao, W., \& Smyth, R. (2011). Economic returns to speaking 'standard mandarin' among migrants in China's urban labour market. Economics of Education Review, 30(2), 342-352. http://dx.doi.org/10.1016/j.econedurev.2010.11.002

Gil, J. (2011). A comparison of the global status of English and Chinese: Towards a new global language? English Today, 27(1), 52-59. http://dx.doi.org/10.1017/S0266078411000149

Hartig, F. (2012). Confucius Institutes and the Rise of China. Journal of Chinese Political Science, 17(1), 53-76. http://dx.doi.org/10.1007/s11366-011-9178-7

Hua, Z., \& Wei, L. (2014), Geopolitics and the Changing Hierarchies of the Chinese Language: Implications for Policy and Practice of Chinese Language Teaching in Britain. The Modern Language Journal, 98, 326-339. http://dx.doi.org/10.1111/j.1540-4781.2014.12064.x

Kirkpatrick, A. (2011). English as an Asian lingua franca and the multilingual model of ELT. Language Teaching, 44(2), 212-224. http://dx.doi.org/10.1017/S0261444810000145

Kobayashi, Y. (2013). Europe versus Asia: foreign language education other than English in Japan's higher education. Higher Education, 66, 269-281. http://dx.doi.org/10.1007/s10734 $-012-9603-7$

Li, D. C. S. (2006). Chinese as a lingua franca in greater China. Annual Review of Applied Linguistics, 26, 149-176. http://dx.doi.org/10.1017/s0267190506000080

Lin, C., Huang, C., \& Chen, C. (2014). Barriers to the adoption of ICT in teaching Chinese as a Foreign language in US universities. ReCALL, 26, 100-116. http://dx.doi.org/10.1017/ S0958344013000268

Lo Bianco, J. (2007). Emergent China and Chinese: Language planning categories. Language Policy, 6(1), 3-26. http://dx.doi.org/10.1007/s10993-006-9042-3

Lu, D. (2008). Pre-imperial Chinese: Its hurdles towards becoming a world language. Journal of Asian Pacific Communication, 18(2), 268-279. http://dx.doi.org/10.1075/japc.18.2.13lu 
Ning, W. (2010). Global English(es) and global Chinese(s): Toward rewriting a new literary history in Chinese. Journal of Contemporary China, 19(63), 159-174. http://dx.doi.org/10.1080/10670560903335884

Pérez-Milans, M. (2015). Mandarin Chinese in London education: Language aspirations in a working-class secondary school. Language Policy, 14(2), 153-181. http://dx.doi.org/ 10.1007/s10993-014-9345-8

Scott, M., \& Tiun, H. (2007). Mandarin-only to mandarin-plus: Taiwan. Language Policy, 6(1), 53-72. http://dx.doi.org/10.1007/s10993-006-9040-5

Smith, R. (2005). Global English: Gift or curse? English Today, 21(2), 56-62. http://dx.doi.org /10.1017/S0266078405002075

Wang, D., \& Adamson, B. (2015). War and Peace: Perceptions of Confucius Institutes in China and USA. The Asia-Pacific Education Researcher, 24(1), 225-234. http://dx.doi.org/ 10.1007/s40299-014-0174-5

Wee, D. (2009). Singapore language enhancer: Identity included. Language and Intercultural Communication, 9(1), 15-23. http://dx.doi.org/10.1080/14708470802684473

Zhang, G. X., \& Linda, M. L. (2010). Chinese language teaching in the UK: present and future. Language Learning Journal, 38(1), 87-97. http://dx.doi.org/10.1080/09571731 003620689

Zhang, Q. (2014). Misinterpretations of intercultural communication among Chinese foreign language educators. Theory and Practice in Language Studies, 4(5), 917-922. http://dx.doi.org/10.4304/tpls.4.5.917-922

Zhang, S. (2011). English as a global language in Chinese context. Theory and Practice in Language Studies, 1(2), 167-176. http://dx.doi.org/10.4304/tpls.1.2.167-176

Zhao, H., \& Huang, J. (2010). China's policy of Chinese as a foreign language and the use of overseas Confucius institutes. Educational Research for Policy and Practice, 9(2), 127-142. http://dx.doi.org/10.1007/s10671-009-9078-1

Zhao, S., \& Liu, Y. (2010). Chinese education in Singapore constraints of bilingual policy from the perspectives of status and prestige planning. Language Problems \& Language Planning, 34(3), 236-258. http://dx.doi.org/10.1075/lplp.34.3.03zha

\section{Copyright Disclaimer}

Copyright for this article is retained by the author(s), with first publication rights granted to the journal.

This is an open-access article distributed under the terms and conditions of the Creative Commons Attribution license (http://creativecommons.org/licenses/by/3.0/). 